\title{
Investigation of partial dislocations fluctuations yields dislocation core parameters.
}

\author{
Pierre-Antoine Geslin \\ Univ. Lyon, INSA-Lyon, MATEIS UMR 5510 CNRS, 69632 Villeurbanne, France \\ E-mail: pierre-antoine.geslin@insa-lyon.fr \\ David Rodney \\ Univ. Lyon, Université Claude Bernard Lyon 1, CNRS, Institut Lumière Matière, \\ LYON, F-69622 France \\ E-mail: david.rodney@univ-lyon1.fr
}

\begin{abstract}
In this work, we investigate the thermal fluctuations of dissociated dislocations in face-centered cubic metals. We first derive an analytical expression of the energy of perturbed interacting partial dislocations. Combining this expression with the equipartition theorem yields a prediction for the thermal fluctuations of interacting partials. Comparing this prediction with atomistic calculations in nickel allows to extract the core energy of the partials at finite temperature and as function of their orientation. We also show how to use these values to parameterize the energetics of perfect dislocations and clarify the scope of validity of representing dissociated dislocations as perfect dislocation lines, an approximation customarily made in dislocation dynamics approaches.
\end{abstract}

\section{Introduction}

The quantitative study of dislocation behavior is central to predict the mechanical behavior of metals and alloys (yield stress, strain hardening, etc.) and to build a quantitative multi-scale simulation framework for crystal plasticity. A key step of this framework consists in transitioning from an atomistic description of the crystal to a dislocation dynamics (DD) model where the positions of the dislocation segments become the degrees of freedom of the system. Describing quantitatively the dislocation energetics and behavior by means of this coarse-grained description is a challenging task.

The energy of a crystal containing a dislocation loop can be divided into two contributions, (i) the elastic energy coming from the stress and strain fields around the dislocation, and (ii) a core energy contribution that is attributed to the defected atoms in the highly distorted core region of the dislocation. If the dislocation is represented with the classical singular elastic theory [1], a small length parameter must be introduced as a cut-off radius around the dislocation core. The non-singular elastic dislocation theory 
[2] also rely on the introduction of a small length parameter representing the spatial spreading distance of the Burgers vector. We note that Peierls-Nabarro type approaches $[3,4,5,6,7]$ and phase-field models for dislocations $[8,9]$ can be considered as nonsingular descriptions where the spreading length-scale is not prescribed but emerges naturally from the model.

In multi-scale frameworks relying on DD methods, the dislocation core energy and core length-scale are input of the coarse-grained DD model and are estimated from atomistic calculations. The most common strategy to estimate these parameters for a specific system consists in performing atomistic simulations of straight dislocations and compare the atomistic energy (obtained either from classical or ab-initio calculations) with the elastic energy of the same dislocation configuration (obtained with a singular or non-singular elastic formalism, with isotropic or anisotropic elasticity depending on the choice of the authors). The difference between the atomistic and elastic contributions is then the dislocation core energy $[10,11,12,13,14,15,16]$. However, a limitation of this approach is that, for straight dislocations, the choice of the core length-scale parameter is arbitrary. Another strategy $[17,16]$ circumvents this limitation by comparing the bow-out of dislocations pinned at their ends between atomistic and non-singular DD simulations. This comparison allows to obtain the core length-scale that matches best the atomistic bow-out. However, the influence of the pinning points on the dislocation shape in atomistic calculations is difficult to assess and to reproduce quantitatively in DD simulations, which may affect the final results. Moreover, in the literature cited above, the atomistic calculations are usually performed at $0 \mathrm{~K}$ and do not yield the core energy at finite temperature (with the exception of Ref. [14]). Finally, most studies extract core parameters for the perfect dislocation while several dislocation problems such as the interaction of a dissociated dislocations with a stacking fault tetrahedron - require to account for the dissociation of the dislocation into partials $[18,19,20]$. It is therefore desirable to extract core parameters for partial dislocations to better parameterize DD models dedicated to these cases [13].

In a recent contribution [21], the authors proposed to apply the capillary fluctuation method $[22,23]$ to dislocations. The objective of this study was two-fold: (i) better characterize the influence of long-range elasticity on the thermal fluctuations that dictate the onset of thermally activated events; (ii) extract quantitative estimates of dislocation parameters at finite temperature (core length-scale, core energy, drag coefficient and inertial mass) to model dislocations quantitatively in higher-scale models such as dislocation dynamics. However, this work was limited to slightly dissociated dislocations as encountered in aluminum. Indeed, for larger dissociation distances, the partials may fluctuate separately, which may not be representative of the behavior of perfect dislocations.

In this work, we generalize the capillary fluctuation method to the case of interacting partial dislocations. We first derive an analytical expression for the interaction energy between two perturbed partials from the non-singular theory of Cai et al. [2]. This allows to apply the equipartition theorem and fit the core line tension parameter to 
reproduce the equilibrium fluctuations obtained in molecular dynamics simulations at different temperatures. Also, when dealing with dislocation microstructures containing a large number of dislocations, most DD models rely on a common approximation: representing dissociated dislocations as perfect dislocation lines [24, 25]. In the limit of small perturbations, we show how to parameterize these simplified DD models in order to best represent the energy of the dissociated dislocation. We also compare our results to the common estimate consisting of neglecting the core energy and choosing the non-singular core parameter arbitrarily (e.g. $a=b$ ).

\section{Energy of perturbed interacting partials dislocations}

The first step of this study consists in deriving an analytical expression for the energy of an arbitrary perturbation of the dissociated dislocation. Fig. 1 presents a sketch of the configuration at hand: two partial dislocations of Burger vectors $\left[b_{s}^{(1)}, b_{e}^{(1)}, 0\right]$ and $\left[b_{s}^{(2)}, b_{e}^{(2)}, 0\right]$, parallel on average to the $\boldsymbol{x}$ direction and separated by an average distance $d$. The position of both partials is described by the contours $C_{1}$ and $C_{2}$. We also assume that the system is $L$-periodic along the $\boldsymbol{x}$ direction and isolated in an infinite elastic medium. We consider also that the distance $d$ is a constant of the problem (e.g. the average position of both partials remains fixed). Therefore, the stacking fault energy of a slightly perturbed state is a constant equal to that of the unperturbed configuration. In the context of thermal fluctuations, this assumption is equivalent to neglecting the so-called breathing mode of the partials [26].

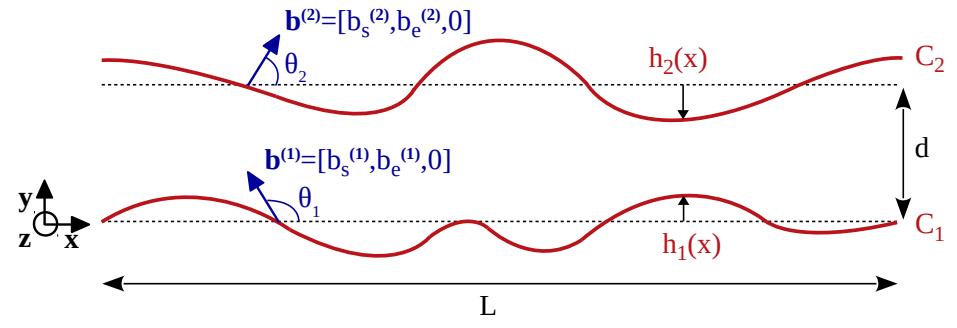

Figure 1. Sketch of perturbed partial dislocations

7

The energy associated with given perturbation on the dislocation of length $L$ can be decomposed as follows:

$$
E_{\text {tot }}=E_{\text {self }}^{(1)}+E_{\text {self }}^{(2)}+E_{\text {int }}^{s-s}+E_{\text {int }}^{e-e}+E_{\text {int }}^{s-e},
$$

where $E_{\text {self }}^{(1)}$ and $E_{\text {self }}^{(2)}$ are the self-energy of the perturbed partial dislocations and the terms denotes $E_{\text {int }}$ refer to the different contributions (screw-screw, edge-edge and screw-edge respectively) of the interaction energy between partials. In the following paragraphs, we will focus on these different contributions and derive closed-form expression for each of them. 


\subsection{Self-energy of a perturbed dislocation}

The energy of the perturbation on an isolated dislocation has been derived in our previous paper [21] and can be divided into a core and an elastic contributions:

$$
E_{\text {self }}^{(i)}=E_{c o}^{(i)}+E_{e l}^{(i)} .
$$

We consider that the contours $C_{1}$ and $C_{2}$ can be described by continuous functions $h_{1}(x)$ and $h_{2}(x)$ (see Fig. 1) and we neglect any perturbation in the $z$ direction. For both partials, the core contribution is expressed as the energy difference between a perturbed and an unperturbed configurations for a section $L$ of the dislocation:

$$
E_{c o}^{(i)}=\int_{0}^{L} \gamma(\tilde{\theta}(x)) \sqrt{1+h_{i}^{\prime}(x)} d x-\int_{0}^{L} \gamma(\theta) d x
$$

where $\tilde{\theta}(x)$ and $\theta$ denotes respectively the local and average dislocation character ( $\theta=0^{\circ}$ for screw and $\theta=90^{\circ}$ for edge orientations) and $\gamma(\theta)$ is the core energy that depends on the dislocation character. Assuming that the functions $h_{i}(x)$ vary slowly (i.e. $\left.\forall x, \quad\left|h_{i}^{\prime}(x)\right| \ll 1\right)$, we derive $[22,21]$

$$
E_{c o}^{(i)}=\Gamma^{(i)}(\theta) \int_{0}^{L} h_{i}^{\prime}(x)^{2} d x
$$

where $\Gamma^{(i)}(\theta)=\gamma^{(i)}(\theta)+\gamma^{(i)^{\prime \prime}}(\theta)$ denotes the core line-tension.

To express Eq. (4) in a compact form, the functions $h_{1}$ and $h_{2}$ are expressed as Fourier series:

$$
\begin{aligned}
& h_{1}(x)=\sum_{n=-N}^{N} C_{n}^{(1)} e^{i k_{n} x} \text { with } \quad C_{-n}^{(1)}=\bar{C}_{n}^{(1)} \\
& h_{2}(x)=\sum_{n=-N}^{N} C_{n}^{(2)} e^{i k_{n} x} \quad \text { with } \quad C_{-n}^{(2)}=\bar{C}_{n}^{(2)}
\end{aligned}
$$

where $k_{n}=2 \pi n / L$ and $C_{n}^{(1)}$ and $C_{n}^{(2)}$ are Fourier coefficients of $h_{1}$ and $h_{2}$ respectively (the overline symbol refers to the complex conjugate). The integer $N$ can be chosen arbitrary large but physically, it is related to the characteristic length of the underlying atomistic lattice (e.g. $N=L / b$ ). With these expressions, we obtained:

$$
E_{c o}^{(i)}=L \Gamma^{(i)}(\theta) \sum_{n=1}^{N} k_{n}^{2}\left|C_{n}\right|^{2} .
$$

In addition to the core contribution, we also account for the elastic term that has been derived in our previous article [21]. The elastic energy is described assuming linear isotropic elasticity and using the non-singular theory of dislocation [2]. The elastic contribution is given by: 


$$
\begin{aligned}
E_{e l}^{(i)}=\frac{\mu L}{2 \pi(1-\nu) a^{2}} \sum_{n=1}^{N_{\max }}\left|C_{n}^{(i)}\right|^{2}[ & -\left(2 b_{s}^{(i)^{2}}(1-\nu)+b_{e}^{(i)^{2}}\right)+a^{2} k_{n}^{2} K_{0}\left(a k_{n}\right)\left(2 b_{s}^{(i)^{2}}-\nu b_{e}^{(i)^{2}}\right) \\
& +a k_{n} K_{1}\left(a k_{n}\right)\left((3-\nu) b_{s}^{(i)^{2}}-b_{e}^{(i)^{2}}\left(1-\frac{a^{2} k_{n}^{2}(1-\nu)}{2}\right)\right) \\
& \left.\left.-a^{2} k_{n}^{2} K_{2}\left(a k_{n}\right)\left(\frac{b_{s}^{(i)^{2}}(1+\nu)}{2}-b_{e}^{(i)}\right)^{2}\right)\right]
\end{aligned}
$$

where $\mu$ and $\nu$ are the isotropic Lamé elastic coefficients and $a$ denotes the core lengthscale parameter of the non-singular theory. The functions $K_{0}, K_{1}$ and $K_{2}$ are the modified Bessel function of the second kind.

\subsection{Interaction between screw components}

The interaction energy between two dislocation loops is given by Eq. (31) of Ref. [2]. Focusing first on the interactions between the screw components, the interaction energy is given by

$$
\begin{aligned}
W_{\text {int }}^{s-s} & =\frac{\mu b_{s}^{(1)} b_{s}^{(2)}}{8 \pi} \int_{C_{1}} \int_{C_{2}} \partial_{k} \partial_{k} R_{a} d l_{x} d l_{x}^{\prime}-\frac{\mu b_{s}^{(1)} b_{s}^{(2)}}{4 \pi(1-\nu)} \int_{C_{1}} \int_{C_{2}} \partial_{1} \partial_{1} R_{a} d l_{x} d l_{x}^{\prime} \\
& +\frac{\mu b_{s}^{(1)} b_{s}^{(2)}}{4 \pi(1-\nu)} \int_{C_{1}} \int_{C_{2}}\left(\partial_{2} \partial_{2} R_{a}+\partial_{3} \partial_{3}\right) d l_{y} d l_{y}^{\prime}
\end{aligned}
$$

with $R_{a}=\sqrt{a^{2}+\left(x^{\prime}-x\right)^{2}+\left(y^{\prime}-y\right)^{2}+\left(z^{\prime}-z\right)^{2}}$. The energy associated with a perturbation is then expressed as the energy difference:

$$
E_{\text {int }}^{s-s}\left(h_{1}, h_{2}\right)=W_{\text {int }}^{s-s}\left(h_{1}, h_{2}\right)-W_{\text {int }}^{s-s}(\mathbf{0}, \mathbf{0})
$$

To simplify the integrals in this expression, we first assume that the perturbations are small:

- $h_{1}(x)$ and $h_{2}(x)$ vary slowly, i.e. $\forall x,\left|h_{1,2}^{\prime}(x)\right|=\mathcal{O}(\epsilon) \ll 1$,

- $h_{1}(x)$ and $h_{2}(x)$ are small compared to $d$, i.e. $\forall x,\left|h_{1,2}(x)\right| / d=\mathcal{O}(\epsilon) \ll 1$,

leading to

$$
\begin{aligned}
& \frac{\left(h_{2}\left(x^{\prime}\right)-h_{1}(x)\right)^{2}}{a^{2}+\left(x^{\prime}-x\right)^{2}+d^{2}}=\mathcal{O}\left(\epsilon^{2}\right) \\
& \frac{d\left(h_{2}\left(x^{\prime}\right)-h_{1}(x)\right)}{a^{2}+\left(x^{\prime}-x\right)^{2}+d^{2}}=\mathcal{O}(\epsilon)
\end{aligned}
$$

Within these limits, the integrands of Eq. (10) can be linearized and further simplified.

We note that in the specific case where $h_{1}=h_{2}$, the first condition of $\forall x,\left|h_{1,2}^{\prime}(x)\right|=$ $\mathcal{O}(\epsilon) \ll 1$ is sufficient to fulfill conditions (11-12). 


$$
\begin{gathered}
E_{\text {int }}^{s-s}=\frac{\mu b_{s}^{(1)} b_{s}^{(2)}}{2 \pi} L \frac{\left(d^{4}+3 a^{2} d^{2}-2 a^{4}\right)}{\left(a^{2}+d^{2}\right)^{3}} \sum_{n=1}^{N}\left(\left|C_{n}^{(1)}\right|^{2}+\left|C_{n}^{(2)}\right|^{2}\right) \\
+\frac{\mu b_{s}^{(1)} b_{s}^{(2)}}{2 \pi(1-\nu)} L \sum_{n=1}^{N}\left(C_{n}^{(1)} \bar{C}_{n}^{(2)}+C_{n}^{(2)} \bar{C}_{n}^{(1)}\right)\left[2 k_{n}^{2} K_{0}\left(\sqrt{a^{2}+d^{2}} k_{n}\right)\right. \\
+\frac{\left((3-\nu)-d^{2} k_{n}^{2}\right) k_{n}}{\sqrt{a^{2}+d^{2}}} K_{1}\left(\sqrt{a^{2}+d^{2}} k_{n}\right) \\
-\frac{\left((6-\nu) d^{2}+\frac{(1+\nu) a^{2}}{2}\right)}{a^{2}+d^{2}} k_{n}^{2} K_{2}\left(\sqrt{a^{2}+d^{2}} k_{n}\right) \\
\left.+\frac{\left(d^{2}+\frac{(1+\nu) a^{2}}{2}\right) d^{2}}{\left(a^{2}+d^{2}\right)^{3 / 2}} k_{n}^{3} K_{3}\left(\sqrt{a^{2}+d^{2}} k_{n}\right)\right]
\end{gathered}
$$

Appendix A provides details for the derivation. We note that this interaction energy is consistent with the expression of the self-energy of a perturbed screw dislocation derived in Ref. [21]. Indeed, considering the same perturbations $\left(C_{n}^{(1)}=C_{n}^{(2)}=C_{n}\right)$ and the same burger vectors $\left(b_{s}^{(1)}=b_{s}^{(2)}=b_{s}\right)$ for both partials, and assuming $d$ small compared to all other distances of the problem $\left(d \ll a\right.$ and $\left.d \ll 1 / k_{n}\right)$, Eq. (13) tends towards:

$$
\begin{aligned}
E_{\text {int }}^{s-s} \underset{d \ll a, 1 / k_{n}}{\simeq} \frac{\mu b_{s}^{2} L}{\pi(1-\nu) a^{2}} \sum_{n=1}^{N}\left|C_{n}\right|^{2}[ & -2(1-\nu)+2 a^{2} k_{n}^{2} K_{0}\left(a k_{n}\right) \\
& \left.+(3-\nu) a k_{n} K_{1}\left(a k_{n}\right)-\frac{1+\nu}{2} a^{2} k_{n}^{2} K_{2}\left(a k_{n}\right)\right]
\end{aligned}
$$

which is exactly twiceł the energy of a perturbation on a screw dislocation from Eq. (8).

\subsection{Interaction between edge components}

We proceed in the same way for the edge-edge interactions. Starting from the interaction energy of two edge dislocations [2]:

$\ddagger$ A self-energy can be defined as half the interaction energy of a dislocation with itself [1]. 


$$
\begin{aligned}
W_{\text {int }}^{e-e} & =\frac{\mu b_{e}^{(1)} b_{e}^{(2)}}{8 \pi} \int_{C_{1}} \int_{C_{2}}\left(\partial_{k} \partial_{k} R_{a}\right) d l_{y} d l_{y}^{\prime} \\
& +\frac{\mu b_{e}^{(1)} b_{e}^{(2)}}{4 \pi(1-\nu)} \int_{C_{1}} \int_{C_{2}}\left(\partial_{1} \partial_{1} R_{a}+\partial_{3} \partial_{3} R_{a}\right) d l_{x} d l_{x}^{\prime} \\
& -\frac{\mu b_{e}^{(1)} b_{e}^{(2)}}{4 \pi(1-\nu)} \int_{C_{1}} \int_{C_{2}}\left(\partial_{2} \partial_{2} R_{a}\right) d l_{y} d l_{y}^{\prime}
\end{aligned}
$$

we follow the same steps as above to obtain the interaction energy of two perturbed edge dislocations:

$$
\begin{aligned}
E_{\text {int }}^{e-e}=\frac{\mu b_{e}^{(1)} b_{e}^{(2)} L}{2 \pi(1-\nu)} \frac{\left(d^{2}-a^{2}\right)}{\left(a^{2}+d^{2}\right)^{2}} \sum_{n=1}^{N}\left(\left|C_{n}^{(1)}\right|^{2}+\left|C_{n}^{(2)}\right|^{2}\right) \\
+\frac{\mu b_{e}^{(1)} b_{e}^{(2)} L}{2 \pi(1-\nu)} \sum_{n=1}^{N}\left(C_{n}^{(1)} \bar{C}_{n}^{(2)}+C_{n}^{(2)} \bar{C}_{n}^{(1)}\right)\left[-\nu k_{n}^{2} K_{0}\left(\sqrt{a^{2}+d^{2}} k_{n}\right)\right. \\
+\frac{\left(\left(a^{2} \frac{1-\nu}{2}+d^{2}\right) k_{n}^{2}-1\right) k_{n}}{\sqrt{a^{2}+d^{2}}} K_{1}\left(\sqrt{a^{2}+d^{2}} k_{n}\right) \\
+\frac{4 d^{2}+a^{2}}{a^{2}+d^{2}} k_{n}^{2} K_{2}\left(\sqrt{a^{2}+d^{2}} k_{n}\right) \\
\left.-\frac{d^{2}}{\sqrt{a^{2}+d^{2}}} k_{n}^{3} K_{3}\left(\sqrt{a^{2}+d^{2}} k_{n}\right)\right]
\end{aligned}
$$

As before, this expression is consistent with the self-energy of an edge dislocation (Eq. (18-19) in the supplemental material of Ref. [21]).

\subsection{Interaction between screw and edge components}

Applying again the same strategy to the screw-edge interaction yields:

$$
\begin{aligned}
E_{\text {int }}^{s-e} & =\frac{\mu\left(b_{s}^{(1)} b_{e}^{(2)}+b_{s}^{(2)} b_{e}^{(1)}\right) L}{2 \pi(1-\nu)} \sum_{n=1}^{N} i\left(C_{n}^{(1)} \bar{C}_{n}^{(2)}-C_{n}^{(2)} \bar{C}_{n}^{(1)}\right)\left[-d k_{n}^{3} K_{0}\left(\sqrt{a^{2}+d^{2}} k_{n}\right)\right. \\
& \left.-\frac{(2-\nu) d k_{n}^{2}}{\sqrt{a^{2}+d^{2}}} K_{1}\left(\sqrt{a^{2}+d^{2}} k_{n}\right)+\frac{d^{3}+\frac{a^{2} d(1+\nu)}{2}}{a^{2}+d^{2}} k_{n}^{3} K_{2}\left(\sqrt{a^{2}+d^{2}} k_{n}\right)\right] .
\end{aligned}
$$

We note that $C_{n}^{(1)} \bar{C}_{n}^{(2)}-C_{n}^{(2)} \bar{C}_{n}^{(1)}$ is a pure imaginary number, hence $E_{\text {int }}^{s-e}$ is real, as expected.

\section{Equipartition theorem and equilibrium fluctuations}

Combining the different interaction terms Eqs. (13),(16) and (17) with the self energy Eqs. (7) and (8) of both partials, the total energy of a perturbed dissociated dislocation can be written as 
$E_{t o t}\left(h_{1}, h_{2}\right)=\sum_{n=1}^{N} a_{n}^{(1)}\left|C_{n}^{(1)}\right|^{2}+a_{n}^{(2)}\left|C_{n}^{(2)}\right|^{2}+b_{n}\left(C_{n}^{(1)} \bar{C}_{n}^{(2)}+C_{n}^{(2)} \bar{C}_{n}^{(1)}\right)+c_{n} i\left(C_{n}^{(1)} \bar{C}_{n}^{(2)}-C_{n}^{(2)} \bar{C}_{n}^{(1)}\right)$

where $a_{n}^{(1)}, a_{n}^{(2)}, b_{n}$ and $c_{n}$ are real numbers which depends on the material parameters $\left(\mu, \nu, d, \boldsymbol{b}^{(1)}, \boldsymbol{b}^{(2)}\right)$ and the wave-vector.

We note that the interaction factors $b_{n}$ and $c_{n}$ are of the same order of magnitude than $a_{n}^{(1)}$ and $a_{n}^{(2)}$ for wave-lengths of the order or larger than the dissociation distance $\left(k_{n} d \sim 1\right)$, stressing the need to account for the interaction energy of the partials to reproduce the correct energy of the system. However, the influence of these interaction terms decreases for negligible wave lengths in the limit $k_{n} d \gg 1$.

The equipartition theorem can not be applied directly to $C_{n}^{(1)}$ and $C_{n}^{(2)}$ because these are not independent quadratic degrees of freedom. To overcome this obstacle, we first write explicitly the true degrees of freedom of the system, that are the real and imaginary parts of the complex amplitudes: $C_{n}^{(1)}=A_{n}^{(1)}+i B_{n}^{(1)}$ and $C_{n}^{(2)}=A_{n}^{(2)}+i B_{n}^{(2)}$. Then, Eq. (18) can be written in the following vectorial form:

$$
E_{\text {tot }}=\sum_{n=1}^{N}\left(A_{n}^{(1)} B_{n}^{(1)} A_{n}^{(2)} B_{n}^{(2)}\right)\left(\begin{array}{cccc}
a_{n}^{(1)} & 0 & b_{n} & c_{n} \\
0 & a_{n}^{(1)} & -c_{n} & b_{n} \\
b_{n} & -c_{n} & a_{n}^{(2)} & 0 \\
c_{n} & b_{n} & 0 & a_{n}^{(2)}
\end{array}\right)\left(\begin{array}{c}
A_{n}^{(1)} \\
B_{n}^{(1)} \\
A_{n}^{(2)} \\
B_{n}^{(2)}
\end{array}\right)=\sum_{n=1}^{N} X_{n}^{T} M_{n} X_{n}
$$

Assuming that the matrices $M_{n}$ are definite positive (in practice, this was shown to be true for all wave-numbers in the case of $\mathrm{Ni}$ ), they can be rewritten using the Cholesky decomposition:

$$
E_{t o t}=\sum_{n=1}^{N} X_{n}^{T} L_{n}^{T} L_{n} X_{n}=\sum_{n=1}^{N} U_{n}^{T} U_{n}
$$

with $U_{n}=L_{n} X_{n}$. The components $u_{n}^{(i)}$ of $U_{n}$ are now independent degrees of freedom, and the equipartition theorem applies:

$$
\begin{aligned}
\forall n, \quad \forall i, \quad\left\langle\left|u_{n}^{(i)}\right|^{2}\right\rangle=k T / 2 \\
\forall n, \quad \forall i \neq j, \quad\left\langle u_{n}^{(i)} u_{n}^{(j)}\right\rangle=0 .
\end{aligned}
$$

Finally, by linear combination, we obtain the average of the Fourier coefficients and predict the amplitudes of the equilibrium perturbations of both partials as a function of temperature: 


$$
\begin{aligned}
& \left\langle\left|C_{n}^{(1)}\right|^{2}\right\rangle=\frac{k T}{2} \sum_{i=1}^{4}\left(L_{1 i}^{-1}\right)^{2}+\left(L_{2 i}^{-1}\right)^{2} \\
& \left\langle\left|C_{n}^{(2)}\right|^{2}\right\rangle=\frac{k T}{2} \sum_{i=1}^{4}\left(L_{3 i}^{-1}\right)^{2}+\left(L_{4 i}^{-1}\right)^{2} .
\end{aligned}
$$

This analytical prediction of the equilibrium fluctuations can now be compared with the fluctuation amplitudes obtained from molecular dynamics simulations.

\section{Molecular dynamics simulations in $\mathrm{Ni}$}

The next step consists in running molecular dynamics (MD) simulations of dislocations to extract the average amplitude of the thermal fluctuations and compare with the theoretical prediction. Concerning the choice of the system, aluminum would be a good candidate because of its elastic isotropy. However, in our previous work [21], we showed that due to the small dissociation distance, spontaneous constriction of the partials occurs even at moderate temperature $(T=300 \mathrm{~K})$. Therefore, the thermal fluctuations in aluminum do not fulfill the condition of Eq. (12), making the analytical treatment proposed in section 2 inadequate. Instead, we will consider nickel that has the advantage of presenting a larger dissociation distance. The Embedded Atom Model (EAM) potential proposed by Mishin et al. [27] was chosen because it reproduces satisfactorily the appropriate elastic constants and stacking fault energy at $0 \mathrm{~K}$ and is therefore expected to yield reliable results for dislocations properties.

In the following, three different temperatures are considered, $450 \mathrm{~K}, 600 \mathrm{~K}$ and $750 \mathrm{~K}$ and simulations are run at zero pressure. These relatively high temperatures are considered in order to limit the influence of lattice friction on the partial dislocations, which is not accounted for in our analysis.

We first compute the temperature dependence of the elastic properties to carefully set the values of elastic constants $\mu$ and $\nu$. After computing the temperature dependence of the lattice parameter (reported in Tab. 1), elastic constants are deduced from NVT runs by averaging the stress arising after a small deformation $\epsilon=10^{-3}$ of a large simulation cell. The standard deviations associated to the measure of the elastic constants are smaller than $1 \mathrm{GPa}$. The estimates of the elastic constants are reported in Tab. 1 together with the cubic anisotropy coefficient $A=2 C_{44} /\left(C_{11}-C_{12}\right)$ and the isotropic moduli obtained from the Bacon-Scattergood average [28, 29]. Note that the significant increase of the elastic constant between 0 and $450 \mathrm{~K}$ is not realistic and is an artifact of the interatomic potential.

In order to characterize precisely the fluctuating properties of partial dislocations, we consider ten different dislocation orientations ranging from screw to edge. In the following, $\phi$ and $\theta$ denote respectively the character of the perfect and partial dislocations. The MD simulations are performed as follows: first we use the software 
Investigation of partial dislocations fluctuations yields dislocation core parameters.

\begin{tabular}{l|c|c|c|c|c|c|c}
$T[\mathrm{~K}]$ & $a_{\text {lat }}[\AA]$ & $C_{11}[\mathrm{GPa}]$ & $C_{12}[\mathrm{GPa}]$ & $C_{44}[\mathrm{GPa}]$ & $A$ & $\mu[\mathrm{GPa}]$ & $\nu$ \\
\hline 0 & 3.52 & 248 & 148 & 125 & 2.49 & 79 & 0.362 \\
450 & 3.5388 & 292 & 173 & 147 & 2.45 & 94 & 0.359 \\
600 & 3.5447 & 287 & 171 & 145 & 2.49 & 92 & 0.361 \\
750 & 3.5507 & 281 & 169 & 141 & 2.52 & 89 & 0.364
\end{tabular}

Table 1. Lattice constant and elastic properties of $\mathrm{Ni}$ [27] as function of temperature. $A=2 C_{44} /\left(C_{11}-C_{12}\right)$ is the cubic anisotropy coefficient.

Babel [30] to introduce a dislocation dipole in a large simulation cell containing between 6 and 7 million atoms depending on the dislocation orientation. For the various dislocation characters, the dimensions of the simulation cells are chosen such that the length of the dislocation, $L \simeq 210 \AA$ is approximately half the distance between the dislocations of the dipole and their periodic images $\left(L_{d} \simeq 420 \AA\right)$. This choice limits the influence of the image dislocations on the fluctuations. After a first relaxation step, the system is equilibrated in the NPT ensemble at the target temperature and zero pressure during 20 ps. The dimensions of the simulation cell are then kept constant during a 100 ps NVT run during which the dislocation fluctuations are recorded. Keeping the same simulation dimensions (especially the length of the dislocation $L$ ) facilitates the analysis of dislocation fluctuations. It is checked that during the NVT part of the simulation, all the components of the average stress tensor remain negligible.

Durin the NVT simulations, the position of the partials is extracted every $0.1 \mathrm{ps}$ using the Dislocation Analysis (DXA) algorithm [31, 32] implemented in Ovito [33]. No post-processing or smoothing of the dislocation line is applied in order to retain the raw serrated dislocation path extracted from DXA. We first wish to check that in the temperature range considered here, the average dissociation distance does not fluctuate significantly in time, as assumed in our analytical approach. Fig. 2 displays the evolution of the dissociation distance as function of the dislocation character at $450 \mathrm{~K}$ and $750 \mathrm{~K}$. The error bars reported in Fig. 2 show one standard deviation of the dissociation distance distribution. Even at $750 \mathrm{~K}$, the standard deviation remains of the order of $5 \%$ of the dissociation distance, which justifies to consider $d$ as a constant.

During the MD runs, we also record the shape of both partials. Fig. 3.a shows as an illustration the position of the partial dislocations (green) and the atoms belonging to the stacking fault (red) for an instantaneous configuration of a $\phi=30^{\circ}$ mixed dislocation at $600 \mathrm{~K}$. From the dislocation lines extracted from DXA, it is straightforward to obtain the equilibrium power spectra of the fluctuations by averaging over a large number of snapshots (in practice 2000) obtained during the MD runs. Fig. 3.b displays the power spectra of both partials of a $30^{\circ}$ mixed dislocation. We note that because of their different characters, the power spectra of the partials differ: the fluctuation amplitude of the $\theta=0^{\circ}$ partial is significantly lower than for $\theta=60^{\circ}$, which is directly visible in Fig. 3.a.

The next step consists in fitting the MD power spectra with the analytical estimate 


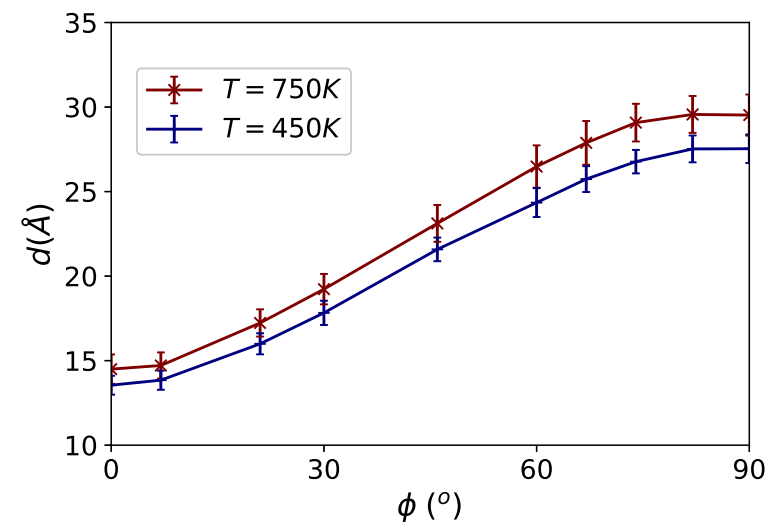

Figure 2. Evolution of the dissociation distance with the character $\phi$ of the perfect dislocation at $450 \mathrm{~K}$ and $750 \mathrm{~K}$.
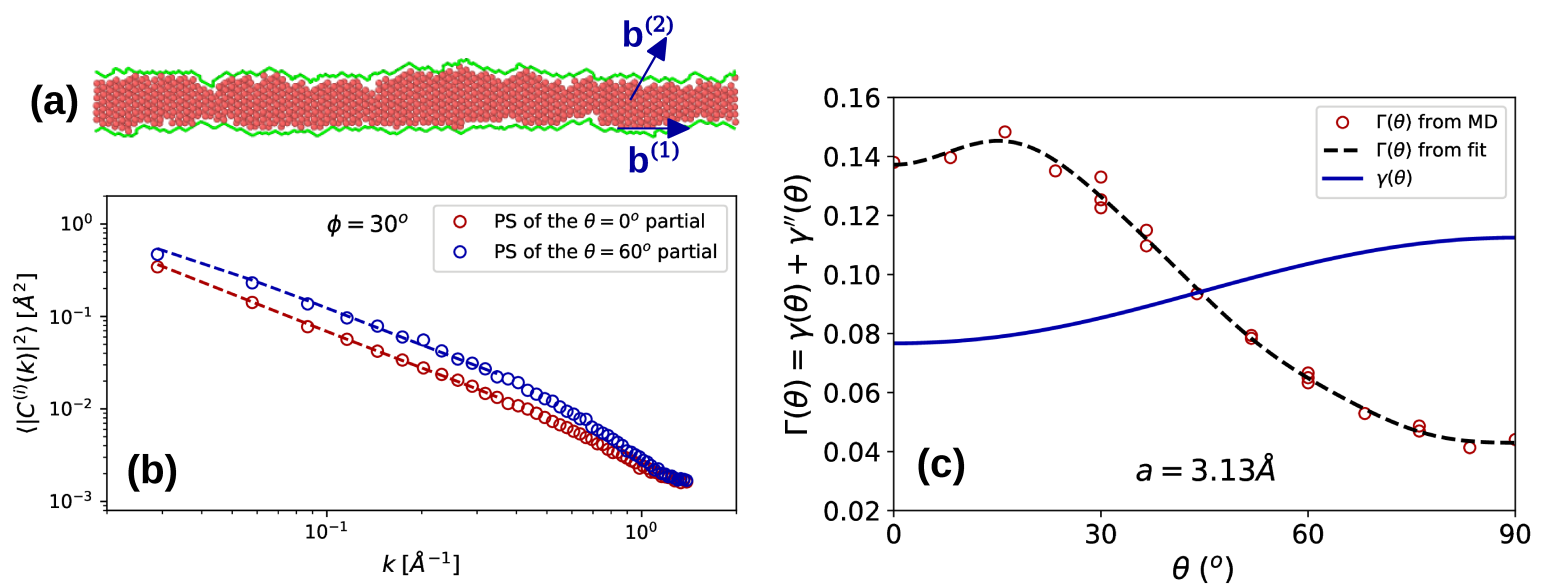

Figure 3. (a) Snapshot of a fluctuating $30^{\circ}$ mixed dislocation at $T=600 \mathrm{~K}$. Atoms belonging to the stacking fault and the partial cores extracted from DXA are shown. (b) Power spectra obtained for both partials (data points) and fits based on the analytical prediction (dashed lines). (c) Core line-tension extracted for various partial dislocation characters (data point) and fit with Eq. (25) (dashed line). The line energy obtained from the fit is shown as a continuous blue line.

of Eqs. (23-24). In order to be consistent, the core parameter should be the same for all partials. Therefore, the power spectra obtained for all partial orientations are fitted against the analytical prediction and the adjustable parameters are the line tensions (which depend on the character) and a unique non-singular core parameter $a$. We restrict ourselves to the long wave-lengths $(\lambda>16 \AA)$ regime because the serrations of the dislocation lines obtained from DXA (see Fig. 3.a) can overestimate the fluctuation spectrum at short wave-lengths. For the $\phi=30^{\circ}$ orientation, the fitted power spectra are shown as dashed lines in Fig. 3.b. We stress the very high quality of the fits obtained for all the orientations.

The fits performed for all the orientations yield a unique fitted parameter $a=3.13 \AA$ 

and a character dependent line-tension $\Gamma(\theta)$ shown in Fig. 3.c at $T=600 \mathrm{~K}$. We note that different perfect dislocations can dissociate to form the same partial character (as an example, the edge and $\phi=60^{\circ}$ mixed dislocations both form a $\theta=30^{\circ}$ partials), which explains why different data points have the same orientation $\theta$ on Fig. 3.c. Our analysis yields very close values of the core line tensions for the same partial orientation, indicating the validity of our approach, and enabling to estimate an uncertainty (of the order of $\pm 5 \%$ ) on the line tensions extracted from the power spectra. This data set is then fitted to a continuous periodic function of the form $\S$ :

$$
\Gamma(\theta)=p_{0}+\sum_{n=1}^{N_{m}} p_{n} \cos (2 n \theta)
$$
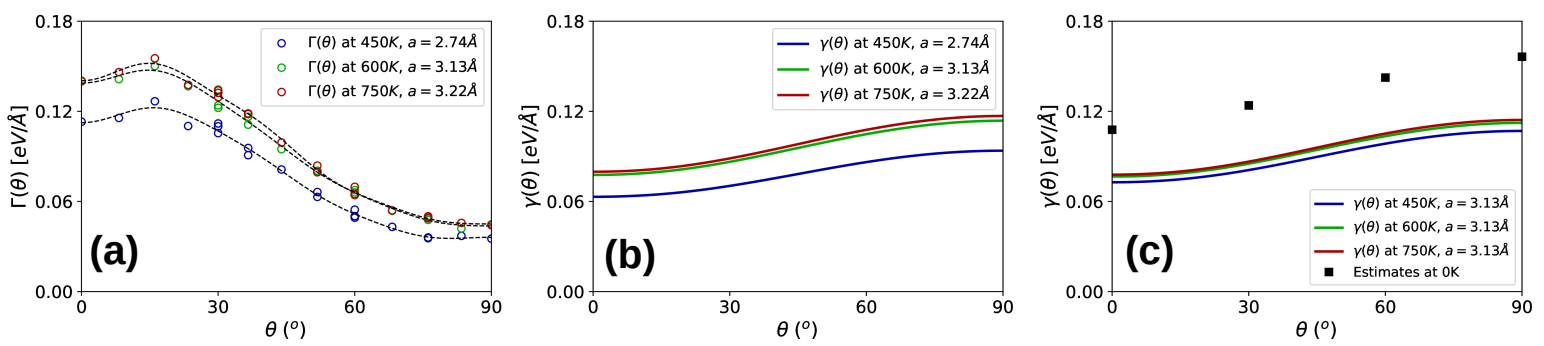

Figure 4. (a) Core line tension as a function of the partial character at different temperatures. (b) Core energy obtained from the fit of panel with best choice of nonsingular parameter. (c) Core energy obtained by fixing the core parameter value to $a=3.13 \AA$.

The number of modes $N_{m}$ can be chosen arbitrarily, but in practice, we used $N_{m}=8$ modes to match the evolution of the data points while avoiding over-fitting. The resulting function is shown as a dash line in Fig. 3.c. From this fit, it is then possible to deduce the line energy $\gamma(\theta)$ (shown with a solid blue line in Fig. 3.c) given by:

$$
\gamma(\theta)=p_{0}+\sum_{n=1}^{N_{m}} \frac{p_{n}}{1-4 n^{2}} \cos (2 n \theta)
$$

to satisfy the relation $\Gamma(\theta)=\gamma(\theta)+\gamma^{\prime \prime}(\theta)$. We note that $\gamma(\theta)$ is extracted at finite temperature and can therefore be referred to as a core free energy. Core line tensions and free energies at various temperatures are shown in Fig. 4.a and Fig. 4.b respectively. We note that the optimal core parameter obtained from the fits increases with temperature, from $a=2.74 \AA$ at $T=450 \mathrm{~K}$ to $a=3.22 \AA$ at $T=750 \mathrm{~K}$, and remains of the order of the lattice spacing, which is consistent with the localized nature of partial dislocation cores.

$\S$ the form is chosen based on the symmetry of $\Gamma(\theta)$ which is necessarily $\pi$-periodic and symmetric with respect to $\theta=0^{\circ}$ and $\theta=90^{\circ}$ 
It is difficult to compare core energies obtained at different temperatures if the non-singular parameters are different (see Fig. 4.b). Indeed, the choice of $a$ controls the amount of line energy carried respectively by the elastic term and the core term; therefore, if $a$ is arbitrarily increased, the line energy coming from the elastic contribution decreases and consequently the core energy has to increase to reproduce the dislocation behavior obtained atomistically. To better compare the core energies at different temperatures, we fit the power spectra with a prescribed value $a=3.13 \AA$. The resulting core free energies are displayed in Fig. 4.c and are very close to one another. This demonstrates that the core contribution does not vary significantly in the temperature range explored here.

In addition, these values of the core energies are compared with estimates obtained at $0 \mathrm{~K}$ from molecular statics. Following our previous work [15], core energies at $0 \mathrm{~K}$ are obtained by comparing the atomistic energy of a relaxed atomistic cell containing a dislocation dipole with the elastic energy obtained from the non-singular representation of dislocations (computed numerically in order to use anisotropic elasticity). Removing the stacking fault contribution estimated as $E_{s f} d$ (where $E_{s f}$ is the stacking fault energy) yields the core energy of partials shown in Fig. 4.c. We find that, the $0 \mathrm{~K}$ estimates fall within $30 \%$ of those obtained from the fluctuations despite the differences between both approaches. The discrepancy between both methods can be attributed to temperature effects and to the approximations used in the molecular statics method: in particular, the fault separating both partials is assumed to be perfect, which may not be accurate because of the evolution of the disregistry close to the partial cores.

\section{Core parameters for perfect dislocations}

In the previous section, we focused on partial dislocations and obtained estimates for their non-singular parameter and core free energy. However, when dealing with lengthscales characteristics of the dislocation microstructure (typically of the order of $1 \mu \mathrm{m}$ ), it become resource-consuming to integrate the dynamics of both partials. For this reason, a common approximation consists in modeling dislocations in FCC crystals as perfect dislocations $[24,34,35]$. Therefore, one may ask if it is possible to derive quantitative parameters for the perfect dislocation based on the knowledge of the partials discussed in the previous section. In the following, the subscript $p a$ (resp. pe) refers to the partial (resp. perfect) description of the dislocation. In this section, we will see how to choose the core parameters $a_{p e}$ and $\Gamma_{p e}(\phi)$, based on the knowledge of $a_{p a}, \Gamma_{p a}(\theta)$ and $d$ (the dissociation distance).

To this end, we compare the energy of a dissociated dislocation slightly perturbed by a sine perturbation $h_{1}(x)=h_{2}(x)=A \sin (k x)$ with the energy of the same perturbation for a perfect dislocation. Before going into the details of this comparison, we must discuss the different length-scales of the system and clarify the domain of validity of the expression derived here. The problem at hand contains three different length-scales, which are the dissociation distance $d$, the wave-length $\lambda=2 \pi / k$ and the amplitude $A$ 
of the perturbation. In this work, we always consider the case of small perturbations $A \ll \lambda$, which is one of the assumptions of our analytical approach. However, the magnitude of the dissociation distance $d$ compared to $A$ and $\lambda$ translates into different situations: Fig. 5.a where $A \ll \lambda<d$ represents a perturbation of small wave-length and small amplitude compared to $d$. In this situation, the partial description is accurate but we will see that using a perfect description leads to inevitable errors. When $A<d<\lambda$ (Fig. 5.b), the interactions between partials become important, but the character of the perfect dislocation does not change significantly, which allows to assume that the stacking fault energy remains unchanged between the unperturbed and perturbed cases. In this situation, both partial and perfect descriptions describe accurately the dislocation energy. In the last case depicted in Fig. 5.c, the dissociation distance is small compared to both the amplitude and length-scale of the perturbation. In this situation, the character of the perfect dislocation changes significantly and so does the dissociation distance which depends on the dislocation character. Therefore, the perturbation leads to an additional energy contribution which comes from changes of stacking fault width along the line, and is negligible in cases (a) and (b). In the following, we will restrict ourselves to the cases (a) and (b) for which the analytical expression derived in section 2 is valid.

(a) A $<<\lambda<$ d

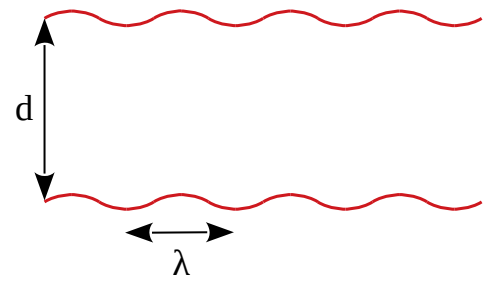

(b) A $<$ d $<\lambda$

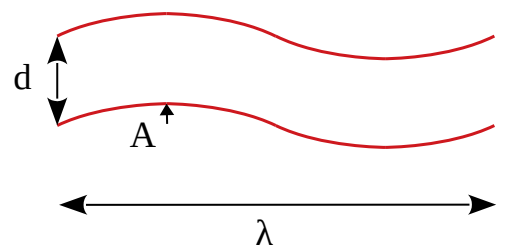

(c) d $<$ A $<<\lambda$

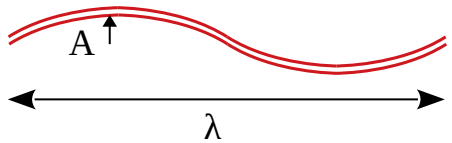

Figure 5. Different ordering of length-scales translate into different situations. of Ref. [21]):

$$
\begin{aligned}
E_{\text {self }}^{e l}\left(\boldsymbol{b}_{p a}^{(i)}\right) & \simeq \frac{\mu b_{p a, s}^{(i)} A^{2} L k^{2}}{16 \pi(1-\nu)}(1+\nu)\left[-\frac{1-\nu}{1+\nu}-\gamma_{e}-\ln \left(\frac{k a_{p a}}{2}\right)\right] \\
& +\frac{\mu b_{p a, e}^{(i)} A^{2} L k^{2}}{16 \pi(1-\nu)}(1-2 \nu)\left[\frac{1}{2}-\gamma_{e}-\ln \left(\frac{k a_{p a}}{2}\right)\right]+\mathcal{O}\left(k^{4} a_{p a}^{4}\right)
\end{aligned}
$$

Similar expressions can be found for the interaction terms between partials in the limit $k \sqrt{a_{p a}^{2}+d^{2}} \rightarrow 0$, i.e. for perturbation wave-lengths large compared to the dissociation distance and the non-singular parameter. 


$$
\begin{aligned}
E_{\text {int }}^{e l}\left(\boldsymbol{b}_{\text {pa }}^{(\mathbf{1})}, \boldsymbol{b}_{\text {pa }}^{(\mathbf{2})}\right) & \simeq \frac{\mu b_{p a, s}^{(1)} b_{p a, s}^{(2)} A^{2} L k^{2}}{16 \pi(1-\nu)}(1+\nu)\left[\frac{(1-\nu)}{(1+\nu)} \frac{d^{4}+a_{p a}^{2} d^{2}-2 a_{p a}^{4}}{\left(a_{p a}^{2}+d^{2}\right)^{2}}-2 \gamma_{e}-2 \ln \left(\frac{k \sqrt{a_{p a}^{2}+d^{2}}}{2}\right)\right] \\
& +\frac{\mu b_{p a, e}^{(1)} b_{p a, e}^{(2)} A^{2} L k^{2}}{16 \pi(1-\nu)}(1-2 \nu)\left[\frac{a_{p a}^{2}+d^{2} /(1-2 \nu)}{a_{p a}^{2}+d^{2}}-2 \gamma_{e}-2 \ln \left(\frac{k \sqrt{a_{p a}^{2}+d^{2}}}{2}\right)\right] \\
& +\mathcal{O}\left(k^{4}\left(a_{p a}^{2}+d^{2}\right)^{2}\right)
\end{aligned}
$$

An interesting (and reassuring) point is that in the limit of vanishing dissociation distance $d \ll a$, we find $E_{\text {int }}^{e l}\left(\boldsymbol{b}_{p}^{(i)}, \boldsymbol{b}_{p}^{(i)}\right)=2 E_{\text {self }}^{e l}\left(\boldsymbol{b}_{p}^{(i)}\right)$, which is consistent with the definition of the self-energy of a dislocation as half the interaction energy with itself [1].

To these elastic terms, we add the core contributions of both partials given by

$$
E_{\text {core }}^{(i)}=A^{2} L k^{2} \frac{\Gamma_{p a}^{(i)}}{4}
$$

where $\Gamma_{p a}^{(i)}$ is the core line tension of partial $(i)$.

We note that in the present case where $h_{1}(x)=h_{2}(x)$, the cross interaction term between the screw and edge components $E_{i n t}^{s-e}\left(b_{s}^{(1)}, b_{s}^{(2)}\right)$ is null. Also, in the limit of small amplitudes (cases (a) and (b) in Fig. 5), the stacking fault does not contribute to the total energy because the area between partials does not change when their shape is modified by a periodic perturbation. Finally, in the limit of large wave-lengths, the total energy of the perturbed partials can be expressed by combining Eqs. (27-29):

$$
\begin{aligned}
E_{p a} & =E_{\text {self }}^{e l}\left(\boldsymbol{b}_{\boldsymbol{p}}^{(\mathbf{1})}\right)+E_{\text {self }}^{e l}\left(\boldsymbol{b}_{\text {pa }}^{(\mathbf{2})}\right)+E_{\text {int }}^{e l}\left(\boldsymbol{b}_{\text {pa }}^{(\mathbf{1})}, \boldsymbol{b}_{\text {pa }}^{(\mathbf{2})}\right)+E_{\text {core }}^{(1)}+E_{\text {core }}^{(2)} \\
& \simeq A^{2} L k^{2}\left[\alpha_{p a}\left(a_{p a}\right)+\beta_{p a} \ln (1 / k)+\frac{\Gamma_{p a}^{(1)}+\Gamma_{p a}^{(2)}}{4}\right]
\end{aligned}
$$

where $\alpha_{p a}$ and $\beta_{p a}$ are coefficients that depend on material parameters but not on the wave-vector. Notably $\alpha_{p}$ depends on the core parameter of the partials while $\beta_{p a}$ does not.

Similarly, the energy of the perturbation of amplitude $A$ of a perfect dislocation can be estimated in the limit of small wave-vectors, yielding a similar expression:

$$
\begin{aligned}
E_{p e} & =E_{\text {self }}^{e l}\left(\boldsymbol{b}_{p e}\right)+E_{p e}^{c o r e} \\
& \simeq A^{2} L k^{2}\left[\alpha_{p e}\left(a_{p e}\right)+\beta_{p e} \ln (1 / k)+\frac{\Gamma_{p e}}{4}\right]
\end{aligned}
$$

We note that based on the expressions of Eq. (27-29), we have $\beta_{p a}=\beta_{p e}$, these parameters being independent of the non-singular parameter. Therefore, to reproduce 
the long-distance behavior of the energy of the dissociated system with a single perfect dislocation, we have the condition:

$$
\Gamma_{p e}=\Gamma_{p a}^{(1)}+\Gamma_{p a}^{(2)}+4\left(\alpha_{p a}\left(a_{p a}\right)-\alpha_{p e}\left(a_{p e}\right)\right)
$$

This relation uniquely defines $\Gamma_{p e}$ as function of $a_{p e}$ and insures that the longrange behavior is correctly reproduced. Looking at Eqs. (31) and (33), one may claim that for very large wave-lengths, the log terms dominate and both expressions are equivalent because $\beta_{p a}=\beta_{p e}$. However, this term grows logarithmically with $1 / k$, and remains of the order of magnitude of the other terms, even for micron-scale wavelengths. Therefore, it is preferable to ensure that Eq. (34) is satisfied in order to model quantitatively dislocations on micro-meter length-scales.

We note that the parameter $a_{p e}$ can be chosen freely to reproduce as accurately as possible the energy of the dissociated dislocation at short or medium wave-lengths. We fit $a_{p e}$ based on all ten orientations and obtain $a_{p e}=6.27 \AA$ from the data obtained at $600 \mathrm{~K}$ for the partials. Fig. 6.a and c displays the fitted energy of the perfect dislocation for screw and edge orientations (blue lines) and compares them with the energy of the partials description (red lines). The error associated with representing the dissociated dislocation as a perfect line is shown with a blue dashed line. A threshold of $20 \%$ error is defined (dashed vertical lines in Fig. 6.a and c) and the corresponding wave-vector is denoted $k_{20 \%}$. We note that the critical wave-vector $k_{20 \%}$ scales like the inverse of the dissociation distance. While the error goes quickly to 0 for $k<k_{20 \%}$, the perfect description fails to reproduce the energy of the perturbation for $k>k_{20 \%}$. The error associated to small wave-lengths is inevitable and shows that using a perfect dislocation representation is hazardous when considering dislocation curvature radii of the order of (or smaller than) $2 \pi / k_{20 \%}$, i.e. about $2 \mathrm{~nm}$ and $7 \mathrm{~nm}$ for the screw and edge dislocations respectively. This small wave-length regime corresponds to the case depicted in Fig. 5.a.

Fig. 6.c and d also shows with black curves the energy of a perturbation when the core parameter is chosen arbitrary (e.g. $a=b$ ) and the core contribution is neglected, which is a common approximation in some dislocation dynamics studies [36, 37]. First, this approach leads to significant errors at small wave-lengths. Especially, the energy of a perturbation becomes negative for small wave-lengths, which explains the divergence of the black curves on Fig. 6.b and 6.d. As explained in Ref. [21], this artifact of the non-singular dislocation theory is overcome by adding a positive core contribution that is predominant at small wave-lengths. In addition, we stress out that the elastic estimate fails to converge towards the appropriate behavior for large wave-lengths. For the edge character, it retains an error as high as $20 \%$ at micron-scale wave-lengths. In dislocation dynamics models, such discrepancy (which comes from neglecting the core contribution) will translate into a systematic error on the critical stresses to escape from pinned configurations.

The curves shown in Fig. 6.a and b rely on the choice of $\Gamma_{p e}$ based on Eq. (34). This can be done for any dislocation character and the evolution of $\Gamma_{p e}$ as a function of the 

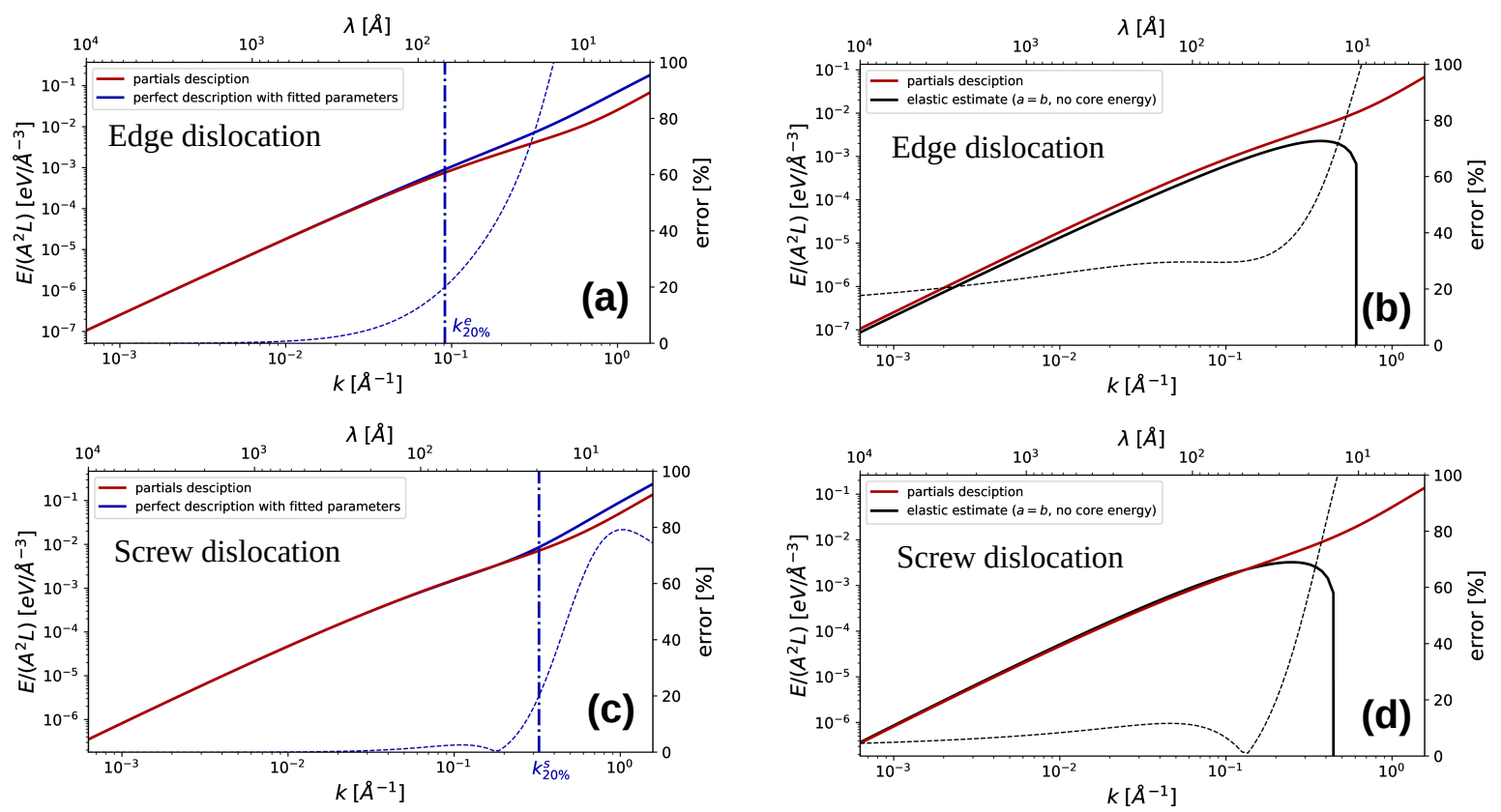

Figure 6. Comparison between different descriptions of the dislocation. (a-b) Comparison between the partial description and the perfect approximation for the screw and edge characters. The energy is shown in log scale with continuous lines while the error associated with the perfect approximation is shown on a linear scale with a dashed line. (c-d) Comparison between the partial description and an elastic estimate of a perfect dislocation with $a=b$ and neglecting the core contribution. Data obtained at $600 \mathrm{~K}$ are used.

perfect dislocation character is shown in Fig. 7. Therefore, the method proposed here yields an optimal choice of parameters $(a$ and $\Gamma(\theta))$ that minimizes the error attributed to the representation of a dissociated dislocation as a single perfect dislocation line. However, we stress out again that these parameters are valid only for small amplitude perturbations as represented in Fig. 5.b.

Now that parameters have been deduced for the perfect dislocation, we can test if the power spectra of the perfect dislocation fluctuations can be predicted by the theoretical estimate derived in Ref. [21] where the core parameters obtained above are used. Fig. 8. a and b displays this comparison for the screw and edge dislocations. In both cases, the predicted fluctuations (dashed curve) matches the fluctuations obtained from MD for wave-vectors smaller than $k_{20 \%}$, i.e. in this limit, representing a dissociated dislocation as a perfect dislocation leads to an acceptable error. However, for wave-vectors greater than $k_{20 \%}$, a large error is associated to the perfect dislocation representation, which explains the growing discrepancy between the predicted power spectrum and the MD results.

The results shown in Fig. 8 demonstrate that for dissociated dislocations, it may be hazardous to extract core parameters from the fluctuations of perfect dislocations. Such strategy requires to consider fluctuations with wave-lengths larger than the dissociation 


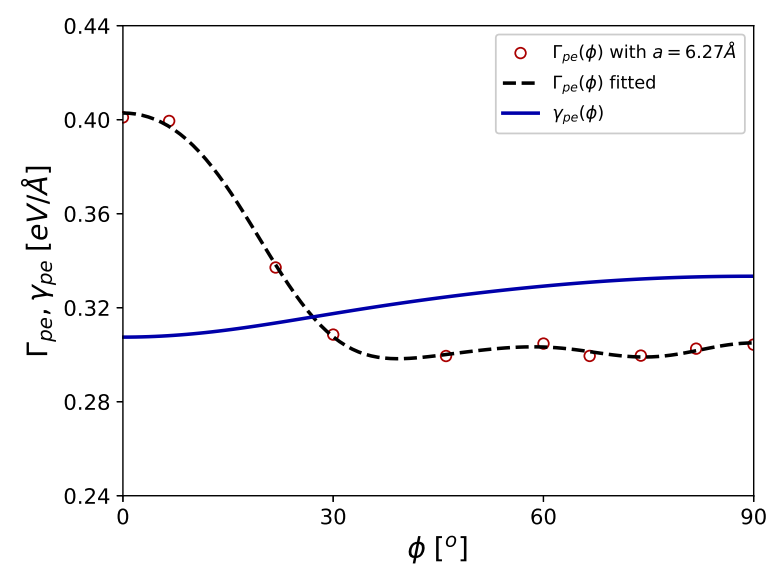

Figure 7. Core energy of the perfect dislocation obtained at $600 \mathrm{~K}$ using Eq. (34) and corresponding line energy (blue line).
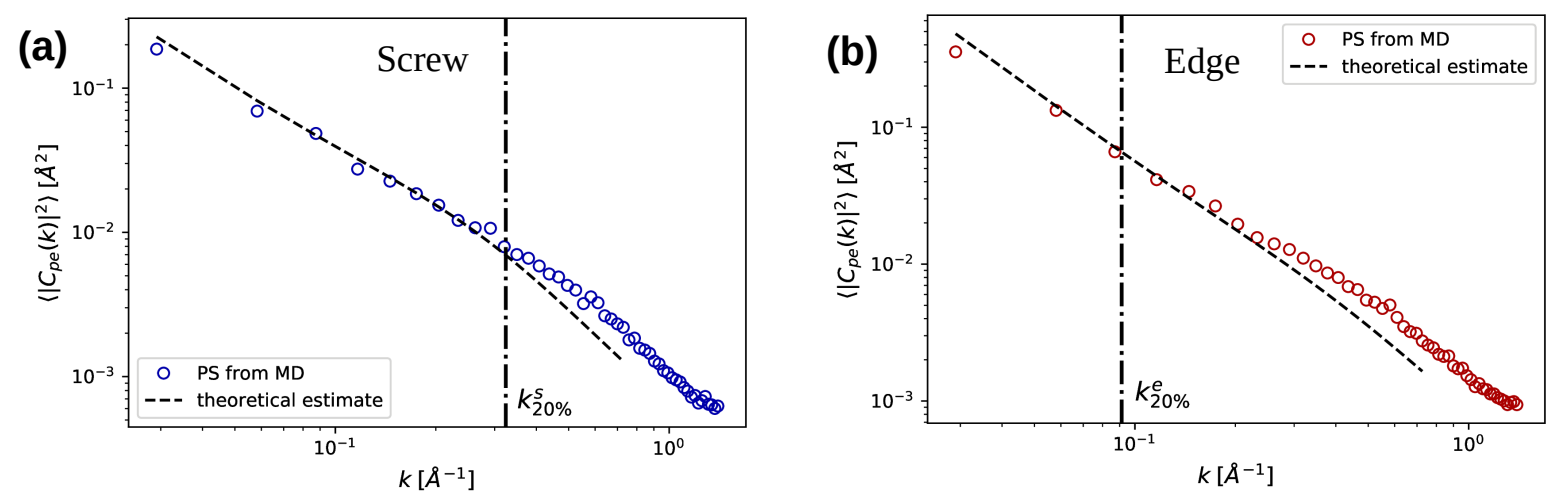

Figure 8. Power spectra of the perfect dislocation obtained from molecular dynamics simulations (symbols) and estimated from theory by considering the fitted core parameters derived above for (a) the screw case and (b) the edge case.

distance, which may be very expensive computationally in the case of FCC metals such as nickel, copper or silver. Therefore, the strategy presented here, which consists in investigating first the partial fluctuations provides an efficient tool to obtain core parameters for perfect dislocations.

\section{Conclusion}

This work builds on our previous contribution [21] in which we investigated the equilibrium fluctuations of a perfect dislocation line and compared with molecular dynamics simulations in aluminum. In the present paper, we extend this work to the case of partial dislocations in FCC metals. We first derive an analytical expression for the energy of interacting partials based on the non-singular dislocation framework of Cai et al. [2]. Based on the equipartition theorem, we derive a prediction for the amplitude 
of the thermal fluctuations of interacting partials. Comparing this analytical prediction with the fluctuations recorded in molecular dynamics simulations in pure nickel yields quantitative estimates for the core parameters (non-singular core radius and core linetension) associated to partials as function of temperature. We note that investigating curved fluctuating dislocations allows to extract an optimal value for the non-singular parameter, in contrast with other approaches focusing on straight dislocations where the non-singular parameter is chosen arbitrarily [10,11, 12, 13, 14, 15]. We also show how the parameters of the partials can be used to determine core parameters for perfect dislocations of various orientations. We note however that these parameters are only valid for small dislocation perturbation. As clarified in section 5, this representation is accurate for wave-lengths larger than a critical distance of the order of the dissociation distance but fails to represent perturbations of short wave-lengths. Finally, we stress out that the classical approximation consisting of neglecting the core contribution and choosing a non-singular parameter $a=b$ leads to non-negligeable errors, even on large micrometer wave-lengths.

The work performed here proposes a rigorous way to obtain core parameters for partial dislocations, enabling to model quantitatively dislocations on small nanometer length-scales in a dislocation dynamics framework that relies on a considerably reduced number of degrees of freedom compared to atomistic calculations. A natural extension of this work would be to investigate atomistic mechanisms such as screw dislocation crossslip with such a quantitative model. Our approach allows to incorporate long-range elastic effects in contrast with previous models where dislocations are described as elastic lines $[38,39,40]$. Another prospect of this work applies to the interaction of dissociated dislocations with a solid solution. In most FCC alloys, the dislocation partials interact with the underlying solutes and roughen [41, 42]. The analytical expression derived here can be used to investigate the interaction of a dissociated dislocation with an underlying solute field and could therefore propose a new treatment of the roughening of a dislocation line and its influence on plastic events. Finally, the analytical treatment proposed here can be generalized to the case of $N$ dislocations interacting elastically. This type of approach would enable to investigate efficiently the collective motion of dislocations in a single slip configuration.

\section{Acknowledgment}

P.A.G wishes to acknowledge the financial support of IDEXLYON project from the University of Lyon in the framework of the Programme Investissements d'Avenir (ANR16-IDEX-005) grant from the University of Lyon IDEX. P.A.G would like to thank Yuanyuan Guo for her support. 


\section{Appendix A. Calculation details for the interaction energy}

The appendix provides more details for the derivation of Eq. (13). Starting from Eq. (9), we consider that the contours $C_{1}$ and $C_{2}$ can be described by continuous functions $h_{1}(x)$ and $h_{2}(x)$. The energy of the perturbation is given by the difference between the energy of the perturbed state and the ground state and can be written as:

$$
\begin{aligned}
E_{\text {int }}^{s-s}\left(h_{1}, h_{2}\right) & =W_{i n t}^{s-s}\left(h_{1}, h_{2}\right)-W_{i n t}^{s-s}(\mathbf{0}, \mathbf{0}) \\
& =-\frac{\mu \nu b_{s}^{(1)} b_{s}^{(2)}}{4 \pi(1-\nu)} \int_{0}^{L} \int_{-\infty}^{+\infty} \frac{1}{\tilde{R}_{a}}-\frac{1}{\bar{R}_{a}} d x d x^{\prime} \\
& -\frac{\mu b_{s}^{(1)} b_{s}^{(2)}}{8 \pi(1-\nu)}\left(2 d^{2}+(1+\nu) a^{2}\right) \int_{0}^{L} \int_{-\infty}^{+\infty} \frac{1}{\tilde{R}_{a}^{3}}-\frac{1}{\bar{R}_{a}^{3}} d x d x^{\prime} \\
& +\frac{\mu b_{s}^{(1)} b_{s}^{(2)}}{4 \pi(1-\nu)} \int_{0}^{L} \int_{-\infty}^{+\infty}\left(\left(x^{\prime}-x\right)^{2}+a^{2}+d^{2}\right)\left(\frac{1}{\tilde{R}_{a}^{3}}-\frac{1}{\bar{R}_{a}^{3}}\right) d x d x^{\prime} \\
& +\frac{\mu b_{s}^{(1)} b_{s}^{(2)}}{2 \pi(1-\nu)} \int_{0}^{L} \int_{-\infty}^{+\infty} \frac{h_{1}^{\prime}(x) h_{2}^{\prime}(x)}{\tilde{R}_{a}} d x d x^{\prime} \\
& -\frac{\mu b_{s}^{(1)} b_{s}^{(2)}}{4 \pi(1-\nu)} \int_{0}^{L} \int_{-\infty}^{+\infty} \frac{\left(h_{2}\left(x^{\prime}\right)-h_{1}(x)\right)^{2} h_{1}^{\prime}(x) h_{2}^{\prime}(x)}{\tilde{R}_{a}^{3}} d x d x^{\prime} \\
& -\frac{\mu b_{s}^{(1)} b_{s}^{(2)}}{4 \pi(1-\nu)} \int_{0}^{L} \int_{-\infty}^{+\infty} d^{2} \frac{h_{1}^{\prime}(x) h_{2}^{\prime}(x)}{\tilde{R}_{a}^{3}} d x d x^{\prime} \\
& -\frac{\mu b_{s}^{(1)} b_{s}^{(2)}}{4 \pi(1-\nu)} \int_{0}^{L} \int_{-\infty}^{+\infty} 2 d \frac{\left(h_{2}\left(x^{\prime}\right)-h_{1}(x)\right) h_{1}^{\prime}(x) h_{2}^{\prime}\left(x^{\prime}\right)}{\tilde{R}_{a}^{3}} d x d x^{\prime}
\end{aligned}
$$

with $\tilde{R}_{a}=\sqrt{a^{2}+\left(x^{\prime}-x\right)^{2}+\left(d+h_{2}\left(x^{\prime}\right)-h_{1}(x)\right)^{2}}$ and $\bar{R}_{a}=\sqrt{a^{2}+\left(x^{\prime}-x\right)^{2}+d^{2}}$.

The next step consists in simplifying the integrals by linearizing the integrand assuming that

$$
\begin{aligned}
\frac{\left(h_{2}\left(x^{\prime}\right)-h_{1}(x)\right)^{2}}{a^{2}+\left(x^{\prime}-x\right)^{2}+d^{2}} & =\mathcal{O}\left(\epsilon^{2}\right) \\
\frac{d\left(h_{2}\left(x^{\prime}\right)-h_{1}(x)\right)}{a^{2}+\left(x^{\prime}-x\right)^{2}+d^{2}} & =\mathcal{O}(\epsilon)
\end{aligned}
$$

Let us focus on $I_{1}$, the integral of Eq. (A.2). It can be further simplified by performing a Taylor expansion, keeping only the lower order terms and discarding the contributions smaller than $\mathcal{O}\left(\epsilon^{2}\right)$ : 


$$
\begin{aligned}
I_{1}= & \int_{0}^{L} \int_{-\infty}^{+\infty} \frac{\left(1+\frac{\left(h_{2}\left(x^{\prime}\right)-h_{1}(x)\right)^{2}}{a^{2}+\left(x^{\prime}-x\right)^{2}+d^{2}}+\frac{2 d\left(h_{2}\left(x^{\prime}\right)-h_{1}(x)\right)}{a^{2}+\left(x^{\prime}-x\right)^{2}+d^{2}}\right)^{-1 / 2}-1}{\sqrt{a^{2}+d^{2}+\left(x^{\prime}-x\right)^{2}}} d x d x^{\prime} \\
= & -\frac{1}{2} \int_{0}^{L} \int_{-\infty}^{+\infty} \frac{\left(h_{2}\left(x^{\prime}\right)-h_{1}(x)\right)^{2}}{\left(a^{2}+d^{2}+\left(x^{\prime}-x\right)^{2}\right)^{3 / 2}} d x d x^{\prime} \\
& -2 d \int_{0}^{L} \int_{-\infty}^{+\infty} \frac{\left(h_{2}\left(x^{\prime}\right)-h_{1}(x)\right)}{\left(a^{2}+d^{2}+\left(x^{\prime}-x\right)^{2}\right)^{3 / 2}} d x d x^{\prime} \\
& +\frac{3 d^{2}}{2} \int_{0}^{L} \int_{-\infty}^{+\infty} \frac{\left(h_{2}\left(x^{\prime}\right)-h_{1}(x)\right)^{2}}{\left(a^{2}+d^{2}+\left(x^{\prime}-x\right)^{2}\right)^{5 / 2}} d x d x^{\prime}+\mathcal{O}\left(\epsilon^{4}\right) .
\end{aligned}
$$

We note that, based on parity arguments, the second integral is null. In the following, the notation $\mathcal{O}\left(\epsilon^{4}\right)$ is omitted for simplicity. Performing similar Taylor expansions of the other integrands and regrouping the different terms yields:

$$
\begin{aligned}
& E_{\text {int }}^{s-s}\left(h_{1}, h_{2}\right)=-\frac{\mu b_{s}^{(1)} b_{s}^{(2)}}{8 \pi(1-\nu)}(3-\nu) \int_{0}^{L} \int_{-\infty}^{+\infty} \frac{\left(h_{2}\left(x^{\prime}\right)-h_{1}(x)\right)^{2}}{\left(a^{2}+\left(x^{\prime}-x\right)^{2}+d^{2}\right)^{3 / 2}} d x d x^{\prime} \\
& +\frac{3 \mu b_{s}^{(1)} b_{s}^{(2)}}{8 \pi(1-\nu)}\left((6-\nu) d^{2}+\frac{(1+\nu)}{2} a^{2}\right) \int_{0}^{L} \int_{-\infty}^{+\infty} \frac{\left(h_{2}\left(x^{\prime}\right)-h_{1}(x)\right)^{2}}{\left(a^{2}+\left(x^{\prime}-x\right)^{2}+d^{2}\right)^{5 / 2}} d x d x^{\prime} \\
& -\frac{15 \mu b_{s}^{(1)} b_{s}^{(2)}}{16 \pi(1-\nu)}\left(2 d^{2}+(1+\nu) a^{2}\right) d^{2} \int_{0}^{L} \int_{-\infty}^{+\infty} \frac{\left(h_{2}\left(x^{\prime}\right)-h_{1}(x)\right)^{2}}{\left(a^{2}+\left(x^{\prime}-x\right)^{2}+d^{2}\right)^{7 / 2}} d x d x^{\prime} \\
& +\frac{\mu b_{s}^{(1)} b_{s}^{(2)}}{2 \pi(1-\nu)} \int_{0}^{L} \int_{-\infty}^{+\infty} \frac{h_{1}^{\prime}(x) h_{2}^{\prime}\left(x^{\prime}\right)}{\sqrt{a^{2}+\left(x^{\prime}-x\right)^{2}+d^{2}}} d x d x^{\prime} \\
& -\frac{\mu b_{s}^{(1)} b_{s}^{(2)}}{4 \pi(1-\nu)} d^{2} \int_{0}^{L} \int_{-\infty}^{+\infty} \frac{h_{1}^{\prime}(x) h_{2}^{\prime}\left(x^{\prime}\right)}{\left(a^{2}+\left(x^{\prime}-x\right)^{2}+d^{2}\right)^{3 / 2}} d x d x^{\prime} .
\end{aligned}
$$

In order to obtain a compact expression of these integrals, we consider that the real functions $h_{1}(x)$ and $h_{2}(x)$ are $L$-periodic and can be written as Fourier series:

$$
\begin{aligned}
& h_{1}(x)=\sum_{n=-N}^{N} C_{n}^{(1)} e^{i k_{n} x} \quad \text { with } \quad C_{-n}^{(1)}=\bar{C}_{n}^{(1)}, \\
& h_{2}(x)=\sum_{n=-N}^{N} C_{n}^{(2)} e^{i k_{n} x} \quad \text { with } \quad C_{-n}^{(2)}=\bar{C}_{n}^{(2)},
\end{aligned}
$$

where $k_{n}=2 \pi n / L$ are the wave-vectors and $C_{n}^{(1)}$ and $C_{n}^{(2)}$ are the Fourier coefficients of $h_{1}$ and $h_{2}$ respectively (the overline symbol refers to the complex conjugate). The integer $N$ can be chosen arbitrary but physically, it is related to the characteristic length of the underlying atomistic lattice (e.g. $N=L / b$ ). Injecting these Fourier series in Eq. (A.12-A.16) yields analytical expression of the different integrals. As an example, let us consider the integral of Eq. (A.12): 


$$
\begin{aligned}
J_{1} & =\int_{0}^{L} \int_{-\infty}^{+\infty} \frac{\left(h_{2}\left(x^{\prime}\right)-h_{1}(x)\right)^{2}}{\left(a^{2}+\left(x^{\prime}-x\right)^{2}+d^{2}\right)^{3 / 2}} d x d x^{\prime} \\
& =\int_{0}^{L} \int_{-\infty}^{+\infty} \frac{\left(\sum_{n=-N}^{N} C_{n}^{(2)} e^{i k_{n} x^{\prime}}-C_{n}^{(1)} e^{i k_{n} x}\right)^{2}}{\left(a^{2}+d^{2}+\left(x^{\prime}-x\right)^{2}\right)^{3 / 2}} d x d x^{\prime} \\
& =\sum_{n=-N}^{N} \sum_{m=-N}^{N} C_{n}^{(1)} C_{m}^{(1)} \int_{0}^{L} \int_{-\infty}^{+\infty} \frac{e^{i\left(k_{n}+k_{m}\right) x}}{\left(a^{2}+d^{2}+\left(x^{\prime}-x\right)^{2}\right)^{3 / 2}} d x d x^{\prime} \\
& +\sum_{n=-N}^{N} \sum_{m=-N}^{N} C_{n}^{(2)} C_{m}^{(2)} \int_{0}^{L} \int_{-\infty}^{+\infty} \frac{e^{i\left(k_{n}+k_{m}\right) x^{\prime}}}{\left(a^{2}+d^{2}+\left(x^{\prime}-x\right)^{2}\right)^{3 / 2}} d x d x^{\prime} \\
& -2 \sum_{n=-N}^{N} \sum_{m=-N}^{N} C_{n}^{(1)} C_{m}^{(2)} \int_{0}^{L} \int_{-\infty}^{+\infty} \frac{e^{i\left(k_{n} x+k_{m} x^{\prime}\right)}}{\left(a^{2}+d^{2}+\left(x^{\prime}-x\right)^{2}\right)^{3 / 2}} d x d x^{\prime}
\end{aligned}
$$

The first sum (Eq. (A.21)) (and similarly the second sum Eq. (A.22)) can be easily computed:

$$
\begin{aligned}
S_{1} & =\sum_{n=-N}^{N} \sum_{m=-N}^{N} C_{n}^{(1)} C_{m}^{(1)} \int_{0}^{L} e^{i\left(k_{n}+k_{m}\right) x}\left(\int_{-\infty}^{+\infty} \frac{d x^{\prime}}{\left(a^{2}+d^{2}+\left(x^{\prime}-x\right)^{2}\right)^{3 / 2}}\right) d x \\
& =\sum_{n=1}^{N} \frac{4 L\left|C_{n}^{(1)}\right|^{2}}{a^{2}+d^{2}} d x
\end{aligned}
$$

because $\int_{0}^{L} e^{i\left(k_{n}+k_{m}\right) x} d x=1$ iff $n=-m$ and 0 otherwise.

The cross product (Eq. (A.23)) can also be reduced and expressed as function of modified Bessel functions of the first kind:

$$
\begin{aligned}
S_{3} & =-2 \sum_{n=-N}^{N} \sum_{m=-N}^{N} C_{n}^{(1)} C_{m}^{(2)} \int_{0}^{L} e^{i\left(k_{n}+k_{m}\right) x}\left(\int_{-\infty}^{+\infty} \frac{e^{i k_{m}\left(x^{\prime}-x\right)}}{\left(a^{2}+d^{2}+\left(x^{\prime}-x\right)^{2}\right)^{3 / 2}} d x^{\prime}\right) d x \\
& =-\sum_{n=1}^{N} \frac{4 L\left(C_{n}^{(1)} \bar{C}_{n}^{(2)}+\bar{C}_{n}^{(1)} C_{n}^{(2)}\right)}{\sqrt{a^{2}+d^{2}}} k_{n} K_{1}\left(\sqrt{a^{2}+d^{2}} k_{n}\right) .
\end{aligned}
$$

Finally, the integral $J_{1}$ can be expressed analytically as function of the Fourier coefficients $C_{n}^{(1)}$ and $C_{n}^{(2)}$ :

$$
J_{1}=4 L \sum_{n=1}^{N} \frac{\left|C_{n}^{(1)}\right|^{2}+\left|C_{n}^{(2)}\right|^{2}}{a^{2}+d^{2}}-\frac{C_{n}^{(1)} \bar{C}_{n}^{(2)}+C_{n}^{(2)} \bar{C}_{n}^{(1)}}{\sqrt{a^{2}+d^{2}}} k_{n} K_{1}\left(\sqrt{a^{2}+d^{2}} k_{n}\right) .
$$

The same steps give: 


$$
\begin{aligned}
& J_{2}=4 L \sum_{n=1}^{N} \frac{2\left(\left|C_{n}^{(1)}\right|^{2}+\left|C_{n}^{(2)}\right|^{2}\right)}{3\left(a^{2}+d^{2}\right)^{2}}-\frac{C_{n}^{(1)} \bar{C}_{n}^{(2)}+C_{n}^{(2)} \bar{C}_{n}^{(1)}}{3\left(a^{2}+d^{2}\right)} k_{n}^{2} K_{2}\left(\sqrt{a^{2}+d^{2}} k_{n}\right), \\
& J_{3}=4 L \sum_{n=1}^{N} \frac{8\left(\left|C_{n}^{(1)}\right|^{2}+\left|C_{n}^{(2)}\right|^{2}\right)}{15\left(a^{2}+d^{2}\right)^{3}}-\frac{C_{n}^{(1)} \bar{C}_{n}^{(2)}+C_{n}^{(2)} \bar{C}_{n}^{(1)}}{15\left(a^{2}+d^{2}\right)^{3 / 2}} k_{n}^{3} K_{3}\left(\sqrt{a^{2}+d^{2}} k_{n}\right) .
\end{aligned}
$$

The fourth integral of Eq. (A.15) can also be expressed as function of modified Bessel functions:

$$
\begin{aligned}
J_{4} & =\int_{0}^{L} \int_{-\infty}^{+\infty} \frac{h_{1}^{\prime}(x) h_{2}^{\prime}\left(x^{\prime}\right)}{\sqrt{a^{2}+\left(x^{\prime}-x\right)^{2}+d^{2}}} d x^{\prime} d x \\
& =-\sum_{n=-N}^{N} \sum_{m=-N}^{N} C_{n}^{(1)} C_{m}^{(2)} k_{n} k_{m} \int_{0}^{L} \int_{-\infty}^{+\infty} \frac{e^{i\left(k_{n} x+k_{m} x^{\prime}\right)}}{\sqrt{a^{2}+\left(x^{\prime}-x\right)^{2}+d^{2}}} d x^{\prime} d x \\
& =-\sum_{n=-N}^{N} \sum_{m=-N}^{N} C_{n}^{(1)} C_{m}^{(2)} k_{n} k_{m} \int_{0}^{L} e^{i\left(k_{n}+k_{m}\right) x}\left(\int_{-\infty}^{+\infty} \frac{e^{i k_{m}\left(x^{\prime}-x\right)} d x^{\prime}}{\sqrt{a^{2}+\left(x^{\prime}-x\right)^{2}+d^{2}}}\right) d x \\
& =2 L \sum_{n=1}^{N}\left(C_{n}^{(1)} \bar{C}_{n}^{(2)}+C_{n}^{(2)} \bar{C}_{n}^{(1)}\right) k_{n}^{2} K_{0}\left(\sqrt{a^{2}+d^{2}} k_{n}\right) .
\end{aligned}
$$

Similarly, we obtain

$$
J_{5}=2 L \sum_{n=1}^{N}\left(C_{n}^{(1)} \bar{C}_{n}^{(2)}+C_{n}^{(2)} \bar{C}_{n}^{(1)}\right) \frac{k_{n}^{3}}{\sqrt{a^{2}+d^{2}}} K_{1}\left(\sqrt{a^{2}+d^{2}} k_{n}\right)
$$

Combining all the different terms yields the following interaction energy for the screw contribution:

$$
\begin{gathered}
E_{i n t}^{s-s}=\frac{\mu b_{s}^{(1)} b_{s}^{(2)}}{2 \pi} L \frac{\left(d^{4}+3 a^{2} d^{2}-2 a^{4}\right)}{\left(a^{2}+d^{2}\right)^{3}} \sum_{n=1}^{N}\left(\left|C_{n}^{(1)}\right|^{2}+\left|C_{n}^{(2)}\right|^{2}\right) \\
+\frac{\mu b_{s}^{(1)} b_{s}^{(2)}}{2 \pi(1-\nu)} L \sum_{n=1}^{N}\left(C_{n}^{(1)} \bar{C}_{n}^{(2)}+C_{n}^{(2)} \bar{C}_{n}^{(1)}\right)\left[2 k_{n}^{2} K_{0}\left(\sqrt{a^{2}+d^{2}} k_{n}\right)\right. \\
+\frac{\left((3-\nu)-d^{2} k_{n}^{2}\right) k_{n}}{\sqrt{a^{2}+d^{2}}} K_{1}\left(\sqrt{a^{2}+d^{2}} k_{n}\right) \\
-\frac{\left((6-\nu) d^{2}+\frac{(1+\nu) a^{2}}{2}\right)}{a^{2}+d^{2}} k_{n}^{2} K_{2}\left(\sqrt{a^{2}+d^{2}} k_{n}\right) \\
\left.+\frac{\left(d^{2}+\frac{(1+\nu) a^{2}}{2}\right) d^{2}}{\left(a^{2}+d^{2}\right)^{3 / 2}} k_{n}^{3} K_{3}\left(\sqrt{a^{2}+d^{2}} k_{n}\right)\right]
\end{gathered}
$$




\section{References}

[1] J.P. Hirth and J. Lothe. Theory of Dislocations. McGraw-Hill, New York, 1968.

[2] W. Cai, A. Arsenlis, C. Weinberger, and V. Bulatov. A non-singular continuum theory of dislocations. J. Mech. Phys. Sol., 54(3):561-587, 2006.

[3] R. Peierls. The size of a dislocation. P. Phys. Soc., 52(1):34, 1940.

[4] F.R.N. Nabarro. Dislocations in a simple cubic lattice. P. Phys. Soc., 59(2):256, 1947.

[5] G. Lu. The Peierls-Nabarro model of dislocations: a venerable theory and its current development. In Handbook of Materials Modeling, pages 793-811. Springer, 2005.

[6] P.-A. Geslin, B. Appolaire, and A. Finel. Investigation of coherency loss by prismatic punching with a nonlinear elastic model. Acta Mater., 71:80-88, 2014.

[7] M. Boleininger, T.D. Swinburne, and S.L. Dudarev. Atomistic-to-continuum description of edge dislocation core: Unification of the Peierls-Nabarro model with linear elasticity. Phys. Rev. Mater., 2(8):083803, 2018.

[8] Y.U. Wang, Y.M. Jin, A.M. Cuitino, and A.G. Khachaturyan. Phase field microelasticity theory and modeling of multiple dislocation dynamics. Appl. Phys. Lett., 78(16):2324-2326, 2001.

[9] D. Rodney, Y. Le Bouar, and A. Finel. Phase field methods and dislocations. Acta Mater., 51(1):17-30, 2003.

[10] J. Li, C.-Z. Wang, J.-P. Chang, W. Cai, V.V. Bulatov, K.-M. Ho, and S. Yip. Core energy and Peierls stress of a screw dislocation in BCC molybdenum: A periodic-cell tight-binding study. Phys. Rev. B, 70(10):104113, 2004.

[11] E. Clouet. Elastic energy of a straight dislocation and contribution from core tractions. Philos. Mag., 89(19):1565-1584, 2009.

[12] E. Clouet, L. Ventelon, and F. Willaime. Dislocation core energies and core fields from first principles. Phys. Rev. Lett., 102:055502, 2009.

[13] E. Martinez, J. Marian, A. Arsenlis, M. Victoria, and J.M. Perlado. Atomistically informed dislocation dynamics in fcc crystals. J. Mech. Phys. Solids, 56(3):869-895, 2008.

[14] X.W. Zhou, R.B. Sills, D.K. Ward, and R.A. Karnesky. Atomistic calculations of dislocation core energy in aluminium. Phys. Rev. B, 95(5):054112, 2017.

[15] P.-A. Geslin, R. Gatti, B. Devincre, and D. Rodney. Implementation of the nudged elastic band method in a dislocation dynamics formalism: application to dislocation nucleation. J. Mech. Phys. Solids, 108:49-67, 2017.

[16] Y. Hu, B.A. Szajewski, D. Rodney, and W.A. Curtin. Atomistic dislocation core energies and calibration of non-singular discrete dislocation dynamics. Modelling and Simulation in Materials Science and Engineering, 28(1):015005, 2019.

[17] B.A. Szajewski, F. Pavia, and W.A. Curtin. Robust atomistic calculation of dislocation line tension. Model. Simul. Mater. Sci. Eng., 23(8):085008, 2015.

[18] E. Martinez, J. Marian, A. Arsenlis, M. Victoria, and J.M. Perlado. A dislocation dynamics study of the strength of stacking fault tetrahedra. part i: interactions with screw dislocations. Phil. Mag., 88(6):809-840, 2008.

[19] E. Martinez, J. Marian, and J.M. Perlado. A dislocation dynamics study of the strength of stacking fault tetrahedra. part ii: interactions with mixed and edge dislocations. Phil. Mag., 88(6):841-863, 2008.

[20] M. Huang and Z. Li. The key role of dislocation dissociation in the plastic behaviour of single crystal nickel-based superalloy with low stacking fault energy: three-dimensional discrete dislocation dynamics modelling. J. Mech. Phys. Sol., 61(12):2454-2472, 2013.

[21] P.-A. Geslin and D. Rodney. Thermal fluctuations of dislocations reveal the interplay between their core energy and long-range elasticity. Phys. Rev. B, 98(17):174115, 2018.

[22] J.J. Hoyt, M. Asta, and A. Karma. Method for computing the anisotropy of the solid-liquid interfacial free energy. Phys. Rev. Lett., 86(24):5530, 2001.

[23] M. Asta, J.J. Hoyt, and A. Karma. Calculation of alloy solid-liquid interfacial free energies from 
atomic-scale simulations. Phys. Rev. B, 66(10):100101, 2002.

[24] B. Devincre, R. Madec, G. Monnet, S. Queyreau, R. Gatti, and L. Kubin. Modeling crystal plasticity with dislocation dynamics simulations: the micromegas code. Mechanics of nanoobjects, pages 81-100, 2011.

[25] R.B. Sills, N. Bertin, A. Aghaei, and W. Cai. Dislocation networks and the microstructural origin of strain hardening. Phys. Rev. Lett., 121(8):085501, 2018.

[26] D. Mordehai, Y. Ashkenazy, I. Kelson, and G. Makov. Dynamic properties of screw dislocations in Cu: a molecular dynamics study. Phys. Rev. B, 67(2):024112, 2003.

[27] Y. Mishin, D. Farkas, M.J. Mehl, and D.A. Papaconstantopoulos. Interatomic Potentials for monoatomic metals from experimental data and ab initio calculations. Phys. Rev. B, 59:3393, 1999.

[28] R.O. Scattergood and D.J. Bacon. The orowan mechanism in anisotropic crystals. Phil. Mag., 31(1):179-198, 1975.

[29] RO Scattergood and DJ Bacon. The strengthening effect of voids. Acta Metallurgica, 30(8):1665$1677,1982$.

[30] E. Clouet. Babel. http://emmanuel.clouet.free.fr/Programs/Babel/, 2010-2016.

[31] A. Stukowski and K. Albe. Extracting dislocations and non-dislocation crystal defects from atomistic simulation data. Model. Simul. Mater. Sci. Eng., 18(8):085001, 2010.

[32] A. Stukowski, V.V. Bulatov, and A. Arsenlis. Automated identification and indexing of dislocations in crystal interfaces. Model. Simul. Mater. Sci. Eng., 20(8):085007, 2012.

[33] A. Stukowski. Visualization and analysis of atomistic simulation data with ovito-the open visualization tool. Model. Simul. Mater. Sci. Eng., 18(1):015012, 2009.

[34] A. Arsenlis, W. Cai, M. Tang, M. Rhee, T. Oppelstrup, G. Hommes, T.G. Pierce, and V.V. Bulatov. Enabling strain hardening simulations with dislocation dynamics. Model. Simul. Mater. Sci. Eng., 15(6):553, 2007.

[35] N. Bertin, M.V. Upadhyay, C. Pradalier, and L. Capolungo. A FFT-based formulation for efficient mechanical fields computation in isotropic and anisotropic periodic discrete dislocation dynamics. Model. Simul. Mater. Sci. Eng., 23(6):065009, 2015.

[36] C. Sobie, L. Capolungo, D.L. McDowell, and E. Martinez. Modal analysis of dislocation vibration and reaction attempt frequency. Acta Mater., 134:203-210, 2017.

[37] R. Santos-Guemes, G. Esteban-Manzanares, I. Papadimitriou, J. Segurado, L. Capolungo, and J. LLorca. Discrete dislocation dynamics simulations of dislocation - $\theta^{\prime}$ precipitate interaction in Al-Cu alloys. J. Mech. Phys. Sol., 118:228-244, 2018.

[38] K. Kang, J. Yin, and W. Cai. Stress dependence of cross slip energy barrier for face-centered cubic nickel. J. Mech. Phys. Solids, 62:181-193, 2014.

[39] A. Malka-Markovitz and D. Mordehai. Cross-slip in face-centered cubic metals: a general Escaig stress-dependent activation energy line tension model. Philos. Mag., 98(5):347-370, 2018.

[40] A. Malka-Markovitz and D. Mordehai. Cross-slip in face centred cubic metals: a general full stress-field dependent activation energy line-tension model. Philos. Mag., 99(12):1460-1480, 2019.

[41] S. Patinet, D. Bonamy, and L. Proville. Atomic-scale avalanche along a dislocation in a random alloy. Phys. Rev. B, 84(17):174101, 2011.

[42] C. Varvenne, A. Luque, and W.A. Curtin. Theory of strengthening in fcc high entropy alloys. Acta Mater., 118:164-176, 2016. 